\title{
Zur Thermodynamik flüssiger Dreistoffgemische
}

\author{
Von Rolf HaAse \\ Aus dem Physikalisch-chemischen Institut der Universität Marburg (Lahn) \\ (Z. Naturforschg. 4a, 342-352 [1949]; eingegangen am 15. November 1948)
}

\begin{abstract}
Es wird eine Übersicht über die wichtigsten Grundgleichungen der Thermodynamik ternärer Flüssigkeitsgemische (ohne Mischungslücke) gegeben. Auf die Bedeutung der von van der Waals und Schreinemakers eingeführten Destillations- und Dampflinien für eine rationelle Darstellung der Gleichgewichte Flüssigkeit-Dampf wird hingewiesen. Die Gesetze für die einfache Destillation von Dreistoffgemischen werden auf moderner Basis abgeleitet. Bisher noch nicht ausgesprochene Sätze über Siedepunktsänderungen bei der Destillation werden aufgestellt, die z.Tl. durch die Messungen von Reinders und de Minjer bestätigt werden. Die von van der Waals, H. A. Lorentz, Schreinemakers und van Dalfsen gefundenen Gesetzmäßigkeiten für den Verlauf der Destillationslinien werden aus thermodynamischen Beziehungen hergeleitet, die auf moderner Begriffsbildung beruhen und daher klar die Voraussetzungen erkennen lassen, unter denen jene Gesetzmäßigkeiten gültig sind: die universellen Grenzgesetze für unendliche Verdünnung und die Gesetze für die idealen Gasgemische im Dampf. Die Diskussion der Destillationslinien wird von vornherein auf den isobaren Fall ausgedehnt und durch eine kurze analytische Behandlung der Dampflinien ergänzt.
\end{abstract}

$\mathrm{I}_{\mathrm{d}=}^{\mathrm{n}}$ n dem Maße, in dem die technischen Prozesse der ,azeotropen“ und ,extraktiven Destillation“ an Bedeutung gewinnen, steigt das Interesse an einer thermodynamischen Theorie der Gleichgewichte in ternären Systemen. Die um die Jahrhundertwende erschienenen experimentellen und theoretischen Arbeiten von Schreinemakers, van der. Waals und van Dalfsen enthalten zwar die wichtigsten grundsätzlichen Erkenntnisse, die sich auf dem Boden der Gibbsschen Thermodynamik gewinnen lassen, sie werden aber bis zum heutigen Tage kaum beachtet, da es ohne sehr eingehende Studien unmöglich ist, die rein thermodynamischen, also allgemeingültigen Resultate von denjenigen Ergebnissen zu trennen, die mit Hilfe der damals allgemein anerkannten van der Waalsschen Zustandsgleichung für flüssige Gemische erhalten worden sind. Es soll in der vorliegenden Arbeit gezeigt werden, wie weit eine von jeglicher Molekulartheorie freie thermodynamische Theorie der flüssigen Dreistoffgemische führt. Naturgemäß erhält man dabei nicht nur neue Formulierungen früher bekannter Resultate, sondern auch einige neue Ergebnisse.

Bezüglich der Herleitung der Grundgleichungen für Mehrstoffsysteme im allgemeinen ${ }^{2,3,6}$ so-

1 W. J ost, Z. Naturforschg. 1, 576 [1946].

2 R. H a a se, Z. Naturforschg. 2a, 492 [1947].

3 R. H a a se, Z. Naturforschg. 3a, 323 [1948]. wie für $Z_{\text {weistoffsysteme }}{ }^{4}$ und Dreistoffsysteme ${ }^{5}$ im besonderen wird auf die früheren Arbeiten verwiesen. Wir beschränken uns hier auf ternäre Systeme ohne Mischungslücke.

Wir setzen außer der Gültigkeit der Grenzgesetze für unendliche Verdünnung, an deren Allgemeinheit nicht gezweifelt werden kann, lediglich die Gesetze idealer Gasmischungen für den Dampf voraus. Will man diese Einschränkung fallen lassen, so muß man die Partialdrucke durch die Fugazitäten (Flüchtigkeiten) ersetzen.

Im ersten Kapitel wird eine Übersicht über die wichtigsten Grundgleichungen der Thermodynamik ternärer Flüssigkeitsgemische gegeben. Im zweiten Kapitel wird über die geometrische Darstellung der Gleichgewichte Flüssigkeit-Dampf berichtet. Im dritten Kapitel erläutern wir den für die rationelle Darstellung der Verdampfungsgleichgewichte und die Theorie der Destillation wichtigen Begriff der ,einfachen Destillation“. Im vierten Kapitel wird die Theorie der Destillationslinien, im fünften die der Dampflinien gegeben.

Die früheren Mitteilungen über Verdampfungsgleichgewichte von Mehrstoffgemischen ${ }^{1} \rightarrow$ werden als I, II, III, IV und V zitiert.

4 R. Haase, Z. Elektrochem.; im Druck.

5 R. Ha ase, Z. Elektrochem., im Druck.

${ }^{6}$ R. Ha a se, Z. Naturforschg. 3a, 285 [1948]. 


\section{1. Úbersicht über die wichtigsten allgemeinen Beziehungen}

Eine Phase aus drei Komponenten, deren $\mathrm{Zu}^{-}$ sammensetzung durch die Molenbrüche $x_{1}$ und $x_{2}$ gekennzeichnet sei, ist dann stabil (absolut stabil oder metastabil), wenn nachfolgende Bedingungen für die Funktion $G$, d. h. die Freie Enthalpie (Gibbssche Freie Energie) je Mol Mischung, gelten $^{5}$ :

$$
\left|\begin{array}{c}
\frac{\partial^{2} G}{\partial x_{1}{ }^{2}} \cdot \frac{\partial^{2} G}{\partial x_{1} \partial x_{2}} \\
\frac{\partial^{2} G}{\partial x_{2} \partial x_{1}} \frac{\partial^{2} G}{\partial x_{2}{ }^{2}}
\end{array}\right|>0, \quad \frac{\partial^{2} G}{\partial x_{1}{ }^{2}}>0,
$$

woraus folgt:

$$
\frac{\partial^{2} G}{d x_{2}{ }^{2}}>0
$$

Werden die $G$-Werte einer Phase durch Lote auf einer $x_{1}, x_{2}$-Ebene angegeben, so bedeutet (1), dafs die $G\left(x_{1}, x_{2}\right)$-Fläche für alle Vertikalschnitte in bezug auf die $x_{1}, x_{2}$-Ebene konvex ist. Bei Gasmischungen ist dies stets der Fall. Bei manchen flüssigen Gemischen ist in bestimmten Temperatur- und Druckbereichen die Bedingung (1) nicht erfüllt; dann ist die Phase absolut instabil (labil) und es tritt Entmischung, d. h. Zerfall in zwei oder drei koexistente Phasen, ein. Die $G$-Fläche weist in diesem Falle sog. „Falten“ auf.

Beim Zweiphasengleichgewicht Flüssigkeit-Dampf müssen die Stabilitätsbedingungen (1) für jede Phase erfüllt sein.

Eine weitere wichtige Beziehung ist die Koexistenzgleichung für das Gleichgewicht FlüssigkeitDampf. Sie lautet bei ternären Gemischen für die Variablen $P$ (Druck), $T$ (Temperatur), $x_{1}$ und $x_{2}$ (Molenbrüche der Komponenten 1 und 2 in der Flüssigkeit), wenn sich die ungestrichenen Größen auf die Flüssigkeit und die gestrichenen auf den Dampf beziehen ${ }^{5}$ :

$$
\begin{array}{rl}
V^{0} d & P-S_{0} d T \\
& =\left\{\left(x_{1}{ }^{\prime}-x_{1}\right) \frac{\partial^{2} G}{\partial x_{1}{ }^{2}}+\left(x_{2}{ }^{\prime}-x_{2}\right) \frac{\partial^{2} G}{\partial x_{1} \partial x_{2}}\right\} d x_{1} \\
& +\left\{\left(x_{1}{ }^{\prime}-x_{1}\right) \frac{\partial^{2} G}{\partial x_{1} \partial x_{2}}+\left(x_{2}{ }^{\prime}-x_{2}\right) \frac{\partial^{2} G}{\partial x_{2}{ }^{2}}\right\} d x_{2},
\end{array}
$$

worin

7 F. A. H. S c h r e in e m a k e r s, Z. physik. Chem. 36, 433 [1901].

8 J. D. van der Waals, Arch. néerl. Sci. exact. natur. (2) 7, 343 [1902], oder Versl. Kon. Akad. Wetensch. Amsterdam 10, 544, 665, 862 [1902]; 11, 88, 224 [1902].

$$
\begin{aligned}
& V_{0}=V^{\prime}-V-\left(x_{1}{ }^{\prime}-x_{1}\right) \frac{\partial V^{\prime}}{\partial x_{1}}-\left(x_{2}{ }^{\prime}-x_{2}\right) \frac{\partial V}{\partial x_{2}}, \\
& S_{0}=S^{\prime}-S-\left(x_{1}{ }^{\prime}-x_{1}\right) \frac{\partial S}{\partial x_{1}}-\left(x_{2}{ }^{\prime}-x_{2}\right) \frac{\partial S}{\partial x_{2}} .
\end{aligned}
$$

Hierin bedeuten $V$ und $S$ das Volumen und die Entropie je Mol Mischung. Eine analoge Gleichung für die Variablen $P, T, x_{1}^{\prime}$ und $x_{2}^{\prime}$ erhält man, wenn man in (2) statt $d x_{1}, d x_{2}, \frac{\partial V}{\partial x_{1}}, \frac{\partial V}{\partial x_{2}}$ usw., $d x_{1}{ }^{\prime}, d x_{2}{ }^{\prime},\left(\frac{\partial V}{\partial r_{1}}\right)^{\prime},\left(\frac{\partial V}{\partial x_{2}}\right)^{\prime}$ usw. setzt.

In genügender Entfernung von kritischen Punkten gilt, wie früher ${ }^{6}$ bewiesen wurde:

$$
V_{0}>0, \quad S_{0}>0 \text {. }
$$

Die Beziehungen (1), (2) und (3) waren schon Schreinemakers ${ }^{7}$ und van der $\mathrm{W}^{4} a \mathrm{ls}^{8}$ bekannt.

Durch Kombination von (1), (2) und (3) läßt sich folgender Satz (Gibbs-Konowalowscher oder 1. Konow a low scher Satz) ableiten $^{\theta}$ : Bei konstanter Temperatur hat der Dampfdruck und bei konstantem Druck der Siedepunkt dann und nur dann ein Extremum, wenn die $\mathrm{Zu}$ sammensetzungen von Flüssigkeit und Dampf gleich sind.

Eine entscheidende Rolle beim Verdampfungsgleichgewicht spielen die "Trennfaktoren" $\alpha_{13}$ und $\alpha_{23}$, die wir folgendermaßen definieren ${ }^{5}$ :

$$
\begin{aligned}
& c_{13} \equiv \frac{x_{1}{ }^{\prime}}{1-x_{1}{ }^{\prime}-x_{2}{ }^{\prime}} / \frac{x_{1}}{1-x_{1}-x_{2}}=\frac{p_{1}{ }^{0}}{p_{3}{ }^{0}} \frac{f_{1}}{f_{3}}, \\
& a_{23} \equiv \frac{x_{2}{ }^{\prime}}{1-x_{1}{ }^{\prime}-x_{2}{ }^{\prime}} / \frac{x_{2}}{1-x_{1}-x_{2}}=\frac{p_{2}{ }^{0}}{p_{3}{ }^{0}} \frac{f_{2}}{f_{3}} .
\end{aligned}
$$

Hierin bedeutet $f_{i}$ den Aktivitätskoeffizienten der Komponente $i$ in der Mischung (Normierung: $\lim _{x_{i} \rightarrow 1} f_{i}=1$ ) und $p_{i}{ }^{0}$ den Dampfdruck der reinen (flüssigen) Komponente $i$.

Ferner setzen wir ${ }^{5}$ :

$$
\begin{aligned}
& \beta_{13} \equiv \frac{\partial \ln a_{13}}{\partial x_{1}}, \quad \gamma_{23} \equiv \frac{\partial \ln a_{23}}{\partial x_{2}}, \\
& \gamma_{13}=\beta_{23} \equiv \frac{\partial \ln a_{23}}{\partial x_{1}}=\frac{\partial \ln a_{13}}{\partial x_{2}} .
\end{aligned}
$$

9 A. Storonkin, Acta physicochim. U.R.S.S. 13, 505 [1940]. Die allgemeinste Formulierung und Ableitung des Satzes von Gibbs und Konowalow (für beliebige bi- und plurivariante Systeme) findet sich bei R. D e f a y, Acad. roy. Belg. (5) 17, 940, 1066 [1937]. S. auch: I. P rigogine u. R. D e f a y, Thermodynamique chimique, Bd. II, S. 388, Liège 1946. 
Wir stellen jetzt die Frage, wie sich der Dampfdruck bzw. Siedepunkt eines ternären Gemischs ändert, wenn eine der drei Komponenten hinzugefügt oder aus dem Gemisch (z. B. durch Rektifikation) entfernt wird. Wenn die Komponente 1 der Flüssigkeit hinzugesetzt oder entzogen wird. so bleibt das Verhältnis der Molenbrüche der beiden anderen Komponenten konstant. Der Punkt im Gibbsschen Darstellungsdreieck (Abb.1), der die Zusammensetzung des Gemischs angibt, bewegt sich also hierbei längs einer durch den Eckpunkt $\mathrm{A}_{1}\left(x_{1}=1\right)$ gehenden Geraden $\mathrm{A}_{1} \mathrm{~N}$, wobei gilt: $\overline{\mathrm{A}_{3} \mathrm{~N}}: \overline{\mathrm{NA}}_{2}=x_{0}: 1-x_{0}$, woraus als Gleichung für die Gerade folgt:

$$
\frac{x_{2}}{1-x_{2}-x_{2}}=\frac{x_{0}}{1-x_{0}}, \text { oder } x_{2}=x_{0}\left(1-x_{1}\right) \text {. }
$$

$x_{0}$ ist der Molenbruch der Komponente 2 in dem von der Komponente 1 freien binären Gemisch (Punkt N).

Für die Druck- bzw. Temperaturänderung ergibt sich für diesen Fall aus Gl. (2), wie an anderer Stelle ${ }^{5}$ abgeleitet wurde $(R=$ Gaskonstante):

$$
\begin{aligned}
& \frac{1}{R T}\left\{V_{0} d P-S_{0} d T\right\} \\
& =\left\{\left(x_{1}{ }^{\prime}-x_{1}\right)\left[\frac{1}{x_{1}\left(1-x_{1}\right)}+\beta_{13}-x_{0} \beta_{23}\right]\right. \\
& \left.\quad+\left(x_{2}{ }^{\prime}-x_{2}\right)\left[\beta_{23}-x_{0} \gamma_{23}\right]\right\} d x_{1} .
\end{aligned}
$$

In (8) sind nur die Vorzeichen von $V_{0}$ und $S_{0}$ infolge (3) allgemein bestimmbar; die $\beta_{i k}$ und $\gamma_{i k}$ können sowohl positiv als auch negativ sein. Daher ist z. B. durch Hinzufügen der Komponente 1 bei konstanter Temperatur sowohl Erhöhung als auch Erniedrigung des Dampfdrucks möglich. Ferner können die Vorzeichen von $\left(d P / d x_{1}\right)_{T}$ und $\left(x_{1}{ }^{\prime}-x_{1}\right)$ in (8) verschieden sein. Damit wird der 2. Konowalowsche Satz bei Dreistoffgemischen (wie überhaupt bei Mehrstoffgemischen) hinfällig ${ }^{5}$.

Ebenso kann gezeigt werden, daß auch der 3. Konowalowsche Satz auf Dreistoffgemische (wie überhaupt auf Mehrstoffgemische) nicht übertragbar ist. Nach diesem Satz ändern sich bei binären Gemischen die Zusammensetzungen von Flüssigkeit und Dampf bei Anderung von Druck bzw. Temperatur im gleichen Sinne?

\section{Darstellung der Verdampfungsgleichgewichte bei ternären Systemen}

Bei binären Systemen wird das Gleichgewicht Flüssigkeit-Dampf entweder im isothermen Dampfdruckdiagramm, das die $P(x)$-Kurven für die Flüssigkeit und den Dampf enthält, oder im isobaren Siedediagramm, das die $T(x)$-Kurven für die Flüssigkeit und den Dampf enthält, dargestellt. Legt man keinen Wert auf die Kenntnis der Dampfdrucke und Siedepunkte, so zeichnet man ein „Gleichgewichtsdiagramm“ für konstante Temperatur oder konstanten Druck, d. h. eine Kurve, die den Molenbruch im Gleichgewichtsdampf als Funktion des Molenbruchs in der Flüssigkeit wiedergibt.

Bei ternären Systemen konstruiert man über dem Gibbsschen Dreieck entweder eine $P\left(x_{1}, x_{2}\right)$ und eine $P\left(x_{1}{ }^{\prime}, x_{2}{ }^{\prime}\right)$-Fläche, d.h. je eine Dampfdruckfläche für die Flüssigkeit und für den Dampf bei gegebener Temperatur, oder eine $T\left(x_{1}, x_{2}\right)$ - und eine $T\left(x_{1}{ }^{\prime}, x_{2}{ }^{\prime}\right)$-Fläche, d. $h$. je eine Siedefläche für die Flüssigkeit und für den Dampf. bei gegebenem Druck. Die Dampfdruckfläche der Flüssigkeit fällt mit der des Dampfes über den drei Eckpunkten und bei binären azeotropen Punkten zusammen; die beiden Flächen berühren einander in ternären azeotropen Punkten, da hier gilt: $\partial P / \partial x_{1}=0, \partial P / \partial x_{2}=0, \partial P / \partial x_{1}{ }^{\prime}=0, \partial P / \partial x_{2}{ }^{\prime}$ $=0, x_{1}=x_{1}{ }^{\prime}, x_{2}=x_{2}{ }^{\prime}$; ein solches Extremum in den Dampfdruckflächen kann ein Maximum, Minimum oder Sattelpunkt sein. Im übrigen liegt die Dampfdruckfläche der Flüssigkeit ganz oberhalb der des Dampfes. Sinngemäß abgeänderte Sätze gelten für die Siedeflächen.

Durch horizontale Ebenen erhält man Paare von Schnittkurven auf den Dampfdruckflächen für bestimmte Drucke bzw. auf den Siedeflächen für bestimmte Temperaturen. Die Projektionen dieser Schnittkurven auf das Darstellungsdreieck ergeben Scharen von Kurvenpaaren, die ,Verdampfungs- und Kondensationskurven" 7 , die im ersten Falle Kurven gleichen Druckes (Isobaren) bei vorgegebener Temperatur, im letzten Falle Kurven gleicher Temperatur (Isothermen) bei vorgegebenem Druck darstellen. Je eine Verdampfungs- und Kondensationskurve beziehen sich auf gleichen Druck und gleiche Temperatur, sind also einander zugeordnet. Die Projektionen derjenigen horizontalen Geraden, die je einen Punkt der Dampfdruck- bzw. Siedefläche der Flüssigkeit mit einem dem Gleichgewichtsdampf ent- 
sprechenden Punkt der Dampfdruck- bzw. Siedefläche des Dampfes verbinden, sind die ,Konnoden"; diese sind also Geraden, die je einen Punkt einer Verdampfungskurve mit einem dem Gleichgewichtsdampf entsprechenden Punkt der zugeordneten Kondensationskurve verbinden.

Legt man wiederum keinen Wert auf die Kenntnis der Dampfdrucke und Siedepunkte, so wird man eine Darstellung wählen, aus der man lediglich die Zuordnung Flüssigkeit-Gleichgewichtsdampf entnehmen kann. Wir denken uns die Dampfdruck- bzw. Siedefläche der Flüssigkeit. Schreiten wir nun auf dieser Fläche in Richtung der Verbindungsgeraden FlüssigkeitspunktDampfpunkt so fort, daß der Dampfpunkt in jedem Augenblick hinter uns liegt, so läßt sich zeigen (s. unten), daß wir uns stets in Richtung abnehmenden Druckes bzw. zunehmender Temperatur bewegen. Die Projektionen dieser Raumkurven auf die $x_{1}, x_{2}$-Ebene haben die Differentialgleichung

$$
\frac{d x_{1}}{d x_{2}}=\frac{x_{1}^{\prime}-x_{1}}{x_{2}^{\prime}-x_{2}}
$$

und werden „Destillationslinien“ genannt. Sie stehen nämlich, wie gezeigt werden wird, in $\mathrm{Zu}$ sammenhang mit dem Prozeß der ,einfachen Destillation“. Die Projektionen derjenigen Raumkurven, auf denen die zugehörigen Dampfpunkte liegen, werden als „Dampflinien“ bezeichnet. Aus Gl. (9) folgt, daß der einem bestimmten Punkt einer Destillationslinie entsprechende Gleichgewichtsdampf durch den Schnittpunkt der an die Destillationslinie in dem betreffenden Punkt gelegten Tangente mit der zugehörigen Dampflinie dargestellt wird. Die durch die Dampflinie abgeschnittenen Tangentenstücke sind aber nichts anderes als die Konnoden. Die Destillationslinien sind also die Enveloppen der Konnoden.

Die Zuordnung Flüssigkeit-Gleichgewichtsdampf kann man daher statt durch ein System von Verdampfungs- und Kondensationskurven nebst Konnoden auch durch die Destillationslinien und die zugehörigen Dampflinien beschreiben. Durch die Destillationslinie wird die Richtung, und durch die Dampflinie die Länge der Strecke Flüssigkeitspunkt-Dampfpunkt angegeben.

\section{Allgemeines über einfache Destillation}

Wird aus einem mit Dampf im Gleichgewicht befindlichen flüssigen Gemisch der Dampf konti- nuierlich entfernt, ohne daß - wie bei der Rektifikation - durch Kondensation ein Rückfluß stattfindet, so spricht man von ,.kontinuierlicher Verdampfung“" oder ,einfacher Destillation“. Es seien $z$ Mole Flüssigkeit vorhanden, aus denen sich $d z$ Mole Dampf bilden. Dann ergibt der Satz der Erhaltung der Masse für jede beliebige Komponente $i$ :

$$
d\left(z x_{i}\right)=x_{i}{ }^{\prime} d z, \text { woraus: } \frac{d z}{z}=\frac{d x_{i}}{x^{\prime}{ }_{i}-x_{i}} .
$$

Da eine solche Gleichung für eine beliebige zweite Komponente $k$ ebenfalls gilt, erhalten wir:

$$
\frac{d x_{i}}{d x_{k}}=\frac{x_{i}^{\prime}-x_{i}}{x_{k}^{\prime}-x_{k}} .
$$

Durch Einführung dieser Beziehung in die allgemeine Koexistenzgleichung [s. II, Gl. (6)] leitet man $a b^{2}$, daß im Falle der einfachen Destillation bei konstanter Temperatur der Dampfdruck monoton sinkt und bei konstantem Druck der Siedepunkt monoton steigt.

Dieser Satz läßt sich für Dreistoffgemische aus den hier angegebenen Gleichungen leicht ableiten. Für $i=1, k=2$ geht Gl. (11) in Gl. (9) über. Wir sehen zunächst, daß die oben definierten ,.Destillationslinien" tatsächlich die behauptete Beziehung zum Prozeß der einfachen Destillation aufweisen. Sie geben die Änderung der Zusammensetzung des flüssigen Rückstandes bei kontinuierlicher Verdampfung eines ternären Gemisches an [vgl. auch III, Gl. (25)]. Die Zusammensetzung des in jedem Augenblick bei der einfachen Destillation aus der Flüssigkeit aufsteigenden Gleichgewichtsdampfes wird nach dem Obigen auf der „Dampflinie“ gefunden, die der betreffenden Destillationslinie zugeordnet ist. Die,,Destillationslinien“ und „Dampflinien" sind in dem anfangs genannten Sinne unter anderer Bezeichnung von van der $\mathrm{W}$ a als ${ }^{8}$ und in dem zuletzt genannten Sinne von Schreinemakers ${ }^{7}$ eingeführt worden.

Kombinieren wir (9) mit (2), so erhalten wir die Differentialgleichung der einfachen Destillation für die Variablen $P, T$ und $x_{1}\left(\operatorname{oder} x_{2}\right)$ :

$$
\begin{gathered}
V_{0} d P-S_{0} d T=\frac{d x_{1}}{x_{1}{ }^{\prime}-x_{1}}\left\{\left(x_{1}{ }^{\prime}-x_{1}\right)^{2} \frac{\partial^{2} G_{2}}{\partial x_{1}{ }^{2}}\right. \\
+2\left(x_{1}{ }^{\prime}-x_{1}\right)\left(x_{2}{ }^{\prime}-x_{2}\right) \frac{\partial^{2} G}{\partial x_{1} \partial x_{2}} \\
\left.+\left(x_{2}{ }^{\prime}-x_{2}\right)^{2} \frac{\partial^{2} G}{\partial x_{2}{ }^{2}}\right\} .
\end{gathered}
$$


Beim kontinuierlichen Verdampfen ist wegen (10) der Ausdruck $d x_{1} /\left(x_{1}{ }^{\prime}-x_{1}\right)$ stets negativ. Beachten wir ferner (3), so bleibt nur das Vorzeichen der in geschweiften Klammern stehenden Summe zu untersuchen. Diese Summe ist aber eine quadratische Form, die wegen (1) positiv definit ist. Somit folgt die oben behauptete Tatsache, daß die Destillationslinien bei konstanter Temperatur im Sinne abnehmenden Druckes und bei konstantem Druck im Sinne steigender Temperatur verlaufen.

Es sei darauf hingewiesen, daß bei der Rektifikation (die praktisch stets bei konstantem Druck ausgeführt wird) der Siedepunkt des Destillationsrückstandes durchaus nicht immer zu steigen braucht. Wegen des Auftretens eines Rücklaufes verliert nämlich Gl. (12) ihre Gültigkeit. Ein häufig realisierter Fall ist der, daß ein Destillat konstanter Zusammensetzung entnommen wird. Bei gegenüber dem Blaseninhalt vernachlässigbar kleinem Kolonneninhalt bewegt sich dann der den Rückstand in der Destillationsblase darstellende Punkt im Gibbsschen Dreieck (s. Abb. 1) auf einer Geraden. Ist das Destillat z. B. die reine Komponente 1, so gilt Gl. (8) mit $d P=0^{*}$. Wie sich aus der Diskussion dieser Gleichung (s.oben) ergibt, kann der Siedepunkt sowohl steigen als auch fallen. Reinders und de Minjer ${ }^{10}$ haben das Fallen des Siedepunktes des Destillationsrückstandes bei der Rektifikation eines heterogenen Gemischs (System Wasser-AcetonChloroform mit reinem Aceton als Destillat) experimentell nachgewiesen. Es folgt aus unseren Ausführungen, daß Sinken der Temperatur im Rückstand schon bei der Rektifikation homogener ternärer Gemische vorkommt.

Bei der einfachen Destillation bezeichnet man als „Destillat“ diejenige Flüssigkeit (mit kontinuierlich veränderlicher Zusammensetzung), die durch totale Kondensation des in jedem Augenblick fortgeführten Gleichgewichtsdampfes entsteht. Wegen der Ungültigkeit des 2. und 3. Satzes von Konowalow bei Dreistoffgemischen kann der Siedepunkt des Destillats bei der isobaren einfachen Destillation steigen oder fallen ${ }^{2}$. Reinders und de Minjer haben das Fallen des Siedepunktes des Destillats innerhalb bestimmter Konzentra-

* Es wird nämlich die Komponente 1 aus der Kolonne entfernt, während die restliche Flüssigkeit in die Blase zurückfließt.

${ }_{10}$ W. Reinders u. C. H. de M injer, Recueil Trav. chim. Pays-Bas 66, 573 [1947]. tionsbereiche nachgewiesen. Es handelte sich dabei um die isobare einfache Destillation des ternären Systems Wasser-Aceton-Chloroform (das eine Mischungslücke aufweist) beim Fortschreiten längs einer (im homogenen Gebiet verlaufenden) Destillationslinie, die durch einen Wendepunkt ausgezeichnet ist.**

\section{Die Destillationslinien}

a) Aufstellung der Differentialg l e i chung

In der Differentialgleichung (9) lassen sich die auf den Dampf bezüglichen Größen $x_{1}{ }^{\prime}$ und $x_{2}{ }^{\prime}$ eliminieren. Aus (4) und (5) leitet man ab:

$\frac{x_{1}{ }^{\prime}-x_{1}}{x_{1}}=\frac{\left(1-x_{1}\right)\left(a_{13}-1\right)-x_{2}\left(a_{23}-1\right)}{1+x_{1}\left(a_{13}-1\right)+x_{2}\left(a_{23}-1\right)}$,
$\frac{x_{2}{ }^{\prime}-x_{2}}{x_{2}}=\frac{\left(1-x_{2}\right)\left(a_{23}-1\right)-x_{1}\left(a_{13}-1\right)}{1+x_{1}\left(a_{13}-1\right)+x_{2}\left(a_{23}-1\right)}$.

Aus (9), (13) und (14) folgt:

$\frac{d x_{1}}{d x_{2}}=\frac{x_{1}}{x_{2}} \frac{\left(1-x_{1}\right)\left(a_{13}-1\right)-x_{2}\left(\alpha_{23}-1\right)}{\left(1-x_{2}\right)\left(a_{23}-1\right)-x_{1}\left(a_{13}-1\right)}$.

Gl. (15) ist die Differentialgleichung der Destillationslinien für die Variablen $x_{1}$ und $x_{2} \cdot \alpha_{13}$ und $\alpha_{\mathrm{s}_{3}}$ sind (außer bei idealen Gemischen, bei denen gilt: $\alpha_{13}=p_{1}{ }^{0} / p_{3}{ }^{0}$ und $\alpha_{23}=p_{2}{ }^{0} / p_{3}{ }^{0}$ ) Funktionen von $x_{1}$ und $x_{2}$, die nach (4) und (5) durch die Konzentrationsabhängigkeit der Aktivitätskoeffizienten gegeben sind. Auch wenn man über diese Abhängigkeit nichts Näheres weiß, kann man ganz allgemeine Gesetzmäßigkeiten aufstellen. Dies soll im folgenden gezeigt werden.

H. A. Lorentz und F. A. H. Schreinemakers ${ }^{\mathbf{1 1}}$ haben den Verlauf der Verdampfungs- und Kondensationskurven in der Nähe von Eckpunkten des Darstellungsdreiecks und von binären azeotropen Punkten untersucht und daraus einige Eigenschaften der Destillationslinien abgeleitet. Van der Waals ${ }^{8}$ ging von Gl. (9) aus und gelangte $\mathrm{zu}$ einer unserer Gl. (15) analogen Beziehung. Van Dalfsen ${ }^{12}$ untersuchte auf diesem Wege die Eigenschaften der Destillationslinien genauer und erhielt Resultate, die über die Lorentz-Schreinemakersschen Rechnungen hinausgehen. Wir werden im wesentlichen - natürlich ohne Bezugnahme auf eine Zustandsgleichung - der Methode von van Dalfsen folgen.

${ }_{11}$ F. A. H. S ch r e i n e make r s, Z. physik. Chem. 36, 413 [1901].

12 B. I. van Dalfsen, Diss. Amsterdam 1906.

** Das bei anderen Systemen nachgewiesene Fallen des Siedepunkts des Destillats bei der Rektifikation ${ }^{13,14}$ steht hiermit nicht in direktem Zusammenhang. 
In den hier gegebenen Abbildungen, die sich auf isobare Destillation beziehen, und bei denen die Reihenfolge der Siedepunkte vermerkt ist, bezeichnen die Pfeile die Richtung der Destillationslinien, also die Richtung steigender Temperatur. Die Eckpunkte sind mit A, B und C bezeichnet. Bei Vergleich mit der Rechnung ist für jeden herausgegriffenen Eckpunkt $A_{3}$, für jeden binären azeotropen Punkt $M$ und für die dazugehörige Dreiecksseite $\mathrm{A}_{1} \mathrm{~A}_{3} \mathrm{zu}$ setzen.

b) Verlauf der Destillationslinien in der Nähe vơn Eckpunkten und Seiten des Darstellungsdreiecks

Für $x_{1} \rightarrow 0$ erhalten wir aus (15): $d x_{1} / d x_{2} \rightarrow 0$, für $x_{2} \rightarrow 0: d x_{1} / d x_{2} \rightarrow \infty$. Daraus ergibt sich: Die Destillationslinien laufen in unmittelbarer Nähe der Dreiecksseiten diesen parallel. Dies ist auch deshalb sofort verständlich, weil die Destillationslinien für die binären Gemische die Dreiecksseiten selbst sind.

Wir betrachten nun den Eckpunkt $A_{3}$ (Abb. 1).

In der Nähe von $A_{3}$ gilt: $x_{1} \rightarrow 0, x_{2} \rightarrow 0$. Aus (15) folgt dann in erster Näherung:

$$
\frac{d r_{1}}{x_{1}}=\frac{d x_{2}}{x_{2}} \frac{a_{13}^{0}-1}{c_{23}^{\prime \prime}-1} .
$$

$\alpha_{13}^{0}$ und $\alpha_{23}^{\gamma}$ sind die Grenzwerte der Trennfaktoren für $x_{3} \rightarrow 1$ [s. V, Gln. (37) und (38)], definiert durch:

$a_{13}^{0} \equiv \frac{K_{13}}{p_{3}^{0}}$,

$$
a_{23}^{0} \equiv \frac{K_{23}}{p_{3}^{0}},
$$

worin $K_{13}$ bzw. $K_{23}$ die auf die Komponente 1 bzw. 2 bezügliche Henrysche Konstante ist.

Integration von (16) gibt:

$$
x_{1}=C x_{2} \frac{a_{13}^{0}-1}{a_{23}^{0}-1} .
$$

$C$ ist die Integrationskonstante, die die einzelnen Kurven der Schar charakterisiert.

Aus (19) ergibt sich:

$$
\frac{d x_{1}}{d x_{2}}=C \frac{a_{13}^{0}-1}{a_{23}^{0}-1} x_{2}^{\frac{a_{13}^{0}-a_{23}^{0}}{a_{23}^{0}-1}}
$$

1. Fall: $\alpha_{13}^{0}>1, \alpha_{23}^{0}>1$. Für $x_{2}=0$ gilt: $x_{1}=0$,

$$
\frac{d x_{1}}{d x_{2}}=\left\{\begin{array}{c}
0 \text { für } a_{13}^{0}>a_{23}^{0}, \\
\infty \text { für } a_{13}^{0}<a_{23}^{0} .
\end{array}\right.
$$

Es wird also diejenige Dreiecksseite (von erster Ordnung) berührt, bei der $\alpha_{i k}^{0}$ den kleineren Wert hat.
Nun gilt gemäß V, Gln. (40) bis (43), für die Steigungen der Dampfdruck- bzw. Siedepunktskurven im Punkte $\mathrm{A}_{3}$ :

$$
\begin{aligned}
& \left(\frac{\partial P}{\partial x_{1}}\right)_{x_{3}=1}=p_{3}^{0}\left(a_{13}^{0}-1\right), \\
& \left(\frac{\partial P}{\partial x_{2}}\right)_{x_{3}=1}=p_{3}^{0}\left(a_{23}^{0}-1\right), \\
& \left(\frac{\partial T}{\partial x_{1}}\right)_{x_{3}=1}=\frac{R T_{3}^{2}}{L_{3}}\left(1-a_{13}^{0}\right), \\
& \left(\frac{\partial T}{\partial x_{2}}\right)_{x_{3}=1}=\frac{R T_{3}^{2}}{L 3}\left(1-a_{23}^{0}\right) .
\end{aligned}
$$

Hierin sind $L_{3}$ und $T_{3}$ Verdampfungswärme und Siedepunkt der Komponente 3.

Aus diesen Beziehungen folgt: Wenn $\alpha_{13}^{0}>1$, $\alpha_{23}>1$ ist, berühren die Destillationslinien in der Nähe eines Eckpunktes im isothermen Falle diejenige Seite, bei der die Steigung der dort beginnenden isothermen Dampfdruckkurve kleiner ist, und im isobaren Falle diejenige Seite, bei der die (negative) Steigung der dort beginnenden isobaren Siedepunktskurve den kleineren Betrag hat.

Für $\alpha_{13}^{0}=\alpha_{23}^{n}$ ergibt sich der Grenzfall: $d x_{1} / d x_{2}$ $=C$, so daß alle Richtungen möglich sind.

2. Fall: $\alpha_{13}^{0}<1, \alpha_{23}^{0}<1$. Für $x_{2}=0$ gilt: $x_{1}=0$,

$$
\frac{d x_{1}}{d x_{2}}=\left\{\begin{array}{l}
0 \text { für } \alpha_{13}^{n}<a_{23}^{0}, \\
\infty \text { für } a_{13}^{n}>a_{23}^{0} .
\end{array}\right.
$$

Es wird mithin im Falle $\alpha_{13}^{0}<1, \alpha_{23}^{n}<1$ diejenige Seite berührt, bei der $\alpha_{i k}^{0}$ den größeren Wert hat, bei der also gemäß (21)-(24) die dort beginnende Dampfdruck- bzw. Siedepunktskurve den kleineren Betrag der Steigung hat. Im Zusammenhang mit dem Vorigen können wir daher sagen: Die Destillationslinien berühren in der Nähe eines Eckpunktes, von dem zwei.steigende oder zwei fallende binäre Dampfdruck- bzw. Siedekurven ausgehen, stets diejenige Seite, bei der die Anfangssteigung der binären Dampfdruck-bzw. Siedekurve kleiner ist.

Dies steht in Übereinstimmung mit dem, was van Dalfsen ${ }^{12}$ für den isothermen Fall fand.

3. Fall: $\alpha_{13}^{0}>1, \alpha_{23}^{0}<1$ oder: $\alpha_{13}^{0}<1, \alpha_{23}^{0}>1$.

Für $x_{2}=0$ gilt jetzt: $x_{1}=\infty$, für $x_{1}=0: x_{2}=\infty$.

Es folgt der für den Sonderfall konstanter Temperatur schon von den älteren Autoren ${ }^{11,12}$ bewiesene Satz: In der Nähe eines Eckpunktes, von dem eine steigende und eine fallende binäre 
Dampfdruck- bzw. Siedekurve ausgehen, haben. die Destillationslinien einen hyperbelartigen Verlauf mit den beiden Dreiecksseiten als Asymptoten (s. Abb. 2 bei C, Abb. 3 bei B und C, Abb. 6 bei A und B).

Dieser hyperbolische Verlauf der Destillationslinien gilt, den Voraussetzungen der Ableitung gemäß, nur für die unmittelbare Nachbarschaft des
Größen bedeuten: $x_{1}=x_{\mathrm{M}}+\xi, x_{2}=\eta$, $\ln \alpha_{13}=\beta_{13}^{\mathrm{M}} \xi+\gamma_{13}^{\mathrm{M}} \eta$, worin

$$
\beta_{13}^{\mathrm{M}}=\left(\frac{\partial \ln \iota_{13}}{\partial x_{1}}\right)_{\mathrm{M}}, \quad \gamma_{13}^{\mathrm{M}}=\left(\frac{\partial \ln a_{13}}{\partial x_{2}}\right)_{\mathrm{M}} .
$$

Aus (15) ergibt sich somit in erster Näherung eine Differentialgleichung, die der von Lorentz und Schreinemakers ${ }^{11}$ aufgestellten und von

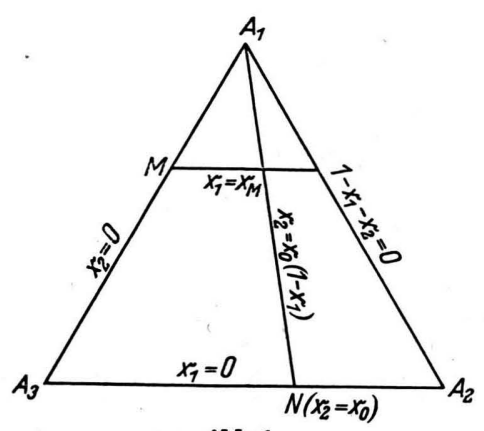

$A b b .1$

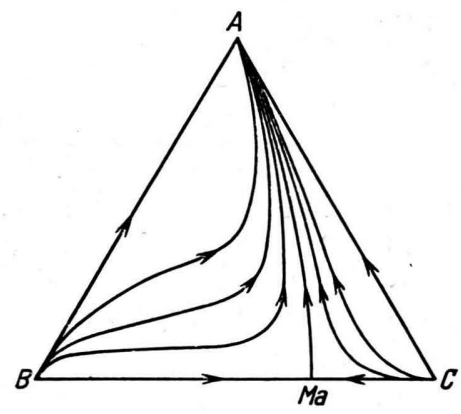

Abb. 4. $A>M a>B$ und $C$

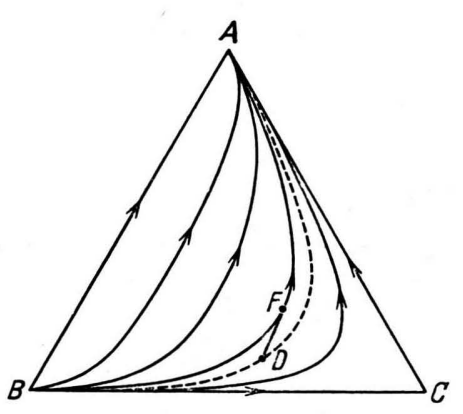

Abb.2. $A>C>B$

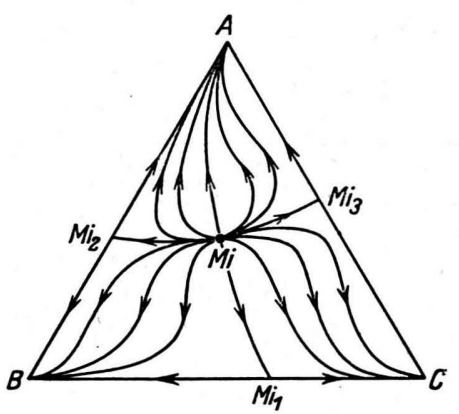

$A b b .5$

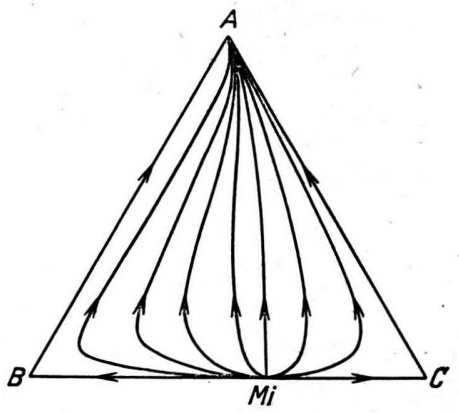

Abb. 3. $A>B$ und $C$

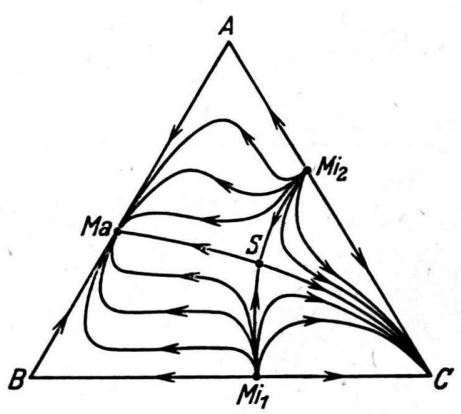

Abb. 6

Abb. 1-6. 1. Das Gibbssche Dreieck. 2. Destillationslinienfeld für ein System ohne azeotropen Punkt. 3. Destillationslinienfeld für ein System mit einem binären azeotropen Punkt (Minimum). 4. Destillationslinienfeld für ein System mit einem binären azeotropen Punkt (Maximum). 5. Destillationslinienfeld für ein System mit drei binären azeotropen Punkten (Minima) und einem ternären azeotropen Punkt (Minimum). 6. Destillationslinienfeld für ein System mit drei binären azeotropen Punkten (2 Minima und 1 Maximum) und einem ternären azeotropen Punkt (Sattelpunkt).

Eckpunktes. An denjenigen Stellen, an denen sich das gegenüber den Dreiecksseiten asymptotische Verhalten der hyperbelartigen Kurven bemerkbar machen würde, haben die Destillationslinien bereits einen ganz anderen Charakter.

c) Verlauf der Destillationslinien in der Nähe von binären und ternären a zeotropen Punkten

Betrachten wir die Nähe des binären azeotropen Punktes M (Abb. 1). Für den Punkt M gilt: $x_{1} \equiv x_{\mathrm{M}}, \quad \alpha_{23} \equiv \alpha_{23}^{\mathrm{M}}, x_{2}=0, x_{1}{ }^{\prime}=x_{1}$, woraus mit (4) folgt: $\alpha_{13}=1$. In unmittelbarer Nachbarschaft von $\mathrm{M}$ können wir ansetzen, wenn $\xi$ und $\eta$ kleine van Dalfsen ${ }^{12}$ näher diskutierten Gleichung mathematisch gleichwertig ist:

$$
\begin{aligned}
& \frac{d \xi}{d \eta}=\frac{\beta_{13}^{\mathrm{M}} x_{\mathrm{M}}\left(1-x_{\mathrm{M}}\right)}{a_{23}^{\mathrm{M}}-1} \frac{\xi}{\eta} \\
& +\frac{\gamma_{13}^{\mathrm{M}} x_{\mathrm{M}}\left(1-x_{\mathrm{M}}\right)-x_{\mathrm{M}}\left(\alpha_{23}^{\mathrm{M}}-1\right)}{\alpha_{23}^{\mathrm{M}}-1} \equiv m \frac{\xi}{\eta}+n .
\end{aligned}
$$

Die Integration liefert:

$$
\begin{aligned}
m \neq 1: \xi & =C \eta^{m}-\frac{n}{m-1} \eta, \\
\frac{d \xi}{d \eta} & =m C \eta^{m-1}-\frac{n}{m-1} ;
\end{aligned}
$$




$$
\begin{aligned}
m=1: \xi & =n \eta \ln \left(C^{\prime} \eta\right), \\
\frac{d \xi}{d \eta} & =n\left[1+\ln \left(C^{\prime} \eta\right)\right] .
\end{aligned}
$$

Für $C=0$ folgt aus (26a):

$$
\xi=-\frac{n}{m-1} \eta \text {. }
$$

Schließt man den Sonderfall $m=1$ aus, so kann man daher sagen: Es gibt stets eine singuläre Kurve, die in der Nähe von $M$ geradlinig verläuft. Diese Kurve werde MN genannt (AMi in Abb. 3, $\mathrm{AMa}$ in Abb. 4, $\mathrm{MiMi}_{1}, \mathrm{MiMi}_{2}$ und $\mathrm{MiMi}_{3}$ in Abb. 5, $\mathrm{SMi}_{1}, \mathrm{SMi}_{2}$ und $\mathrm{SMa}$ in Abb. 6).

Die Bedingung $C \neq 0$ gibt den Verlauf aller übrigen Destillationslinien in der Nähe von $M$. Es sind dabei folgende Fälle $\mathrm{zu}$ unterscheiden:

A. $m>0$. Wir betrachten $(26 \mathrm{~b})$.

1. $m>1: \lim _{\eta \rightarrow 0} \frac{d \xi}{d \eta}=-\frac{n}{m-1}$.

Es wird die ausgezeichnete Kurve MN berührt (in Abb. 6 bei $\mathrm{Mi}_{1}$ und $\mathrm{Mi}_{2}$ ).

$$
\text { 2. } m<1: \lim _{\eta \rightarrow 0} \frac{d \xi}{d \eta}= \pm \infty \text {. }
$$

Es wird die Seite $A_{1} A_{2}$ berührt (in Abb. 3 bei $\mathrm{Mi}$, in Abb. 6 bei Ma).

3. $m=1$ : Dies ist ein Sonderfall, der zwischen den beiden vorigen liegt und bei dem die Gln. (27a) und $(27 \mathrm{~b})$ gelten. Die singuläre Kurve $\left(C^{\prime}=0\right)$ fällt mit der Seite $A_{1} A_{2}$ zusammen.

B. $m<0$. Es folgt aus $(26 \mathrm{a})$

für $\eta=0: \xi= \pm \infty$, für $\eta=\infty: \xi=-\frac{n}{m-1} \eta$.

Die Destillationslinien haben einen hyperbelartigen Verlauf mit $\mathrm{MN}$ und $\mathrm{MA}_{1}$ bzw. MN und $\mathrm{MA}_{3}$ als Asymptoten (in Abb. 4 bei Ma, in Abb. 5 bei $\mathrm{Mi}_{1}, \mathrm{Mi}_{2}$ und $\mathrm{Mi}_{2}$ ). In diesem Falle heißt die ausgezeichnete Kurve MN ,Grenzlinie“, weil sie die Scharen der Destillationslinien in zwei Bündel und dadurch das Darstellungsdreieck in zwei „Destillationsfelder" teilt.

Legen wir uns jetzt die Frage vor, wann die einzelnen Fälle eintreten. Fall B liegt nach (25) vor, wenn

$$
\begin{aligned}
\beta_{13}^{\mathrm{M}}>0, & \alpha_{23}^{\mathrm{M}}<1, \\
\operatorname{oder} \beta_{13}^{\mathrm{M}}<0, & \alpha_{23}^{\mathrm{M}}>1 \text { ist. }
\end{aligned}
$$

Nun gilt gemäß IV, Gì. (49), für die Krümmung der isothermen Dampfdruckkurve am azeotropen
Punkt M für das binäre Gemisch 1,3:

$$
\left(\frac{\partial^{2} P}{\partial x_{1}^{2}}\right)_{\mathrm{M}}=\beta_{13}^{\mathrm{Y}} P_{\mathrm{M}}\left[1+x_{\mathrm{M}}\left(1-x_{\mathrm{M}}\right) \beta_{13}^{\mathrm{M}}\right] \text {. }
$$

Hierin bedeutet $P_{\mathrm{M}}$ den Extremdruck für das binäre Gemisch. Der in eckigen Klammern stehende Ausdruck ist infolge der Stabilitätsbedingung [s. IV, Gln. (8) und (17)] stets positiv. $\beta_{13}^{\text {MI }}>0$ entspricht also einem Dampfdruckminimum und somit einem Siedepunktsmaximum, $\beta_{13}^{\mathrm{r}}<0$ einem Dampfdruckmaximum bzw. Siedepunktsminimum für das binäre Gemisch 1,3.

Ferner erhalten wir gemäß V, Gln. (45) bzw. (46), für die Steigung der Schnittkurve, die durch eine auf der Geraden $x_{1}=x_{M}$ (s. Abb.1) senkrecht errichtete Ebene auf der Dampfdruck- bzw. Siedefläche erzeugt wird, im Punkte $M$ folgende Ausdrücke:

$$
\begin{aligned}
& \left(\frac{\partial P}{\partial x_{2}}\right)_{\mathrm{M}}=P_{\mathrm{M}}\left(\alpha_{23}^{\mathrm{M}}-1\right), \\
& \left(\frac{\partial T}{\partial x_{2}}\right)_{\mathrm{M}}=\frac{R T_{\mathrm{M}}^{2}}{L_{\mathrm{M}}^{2}}\left(1-\alpha_{23}^{\mathrm{u}}\right) .
\end{aligned}
$$

$L_{\mathrm{M}}$ ist die Verdampfungswärme des binären azeotropen Gemischs bei der Extremtemperatur $T_{\mathrm{M}}$. Es entspricht also z. B. $\alpha_{23}^{\mathrm{M}}<1$ einem Fallen der betreffenden Schnittkurve auf der Dampfdruckfläche im Punkte $M$ bzw. einem Steigen der Schnittkurve auf der Siedefläche im Punkte M.

Im isobaren Diagramm wird daher Fall B dann zu erwarten sein, wenn ein binäres Siedepunktsmaximum demjenigen Eckpunkt gegenüber liegt, der der Komponente mit dem höchsten Siedepunkt entspricht (Abb.4), und wenn ein binäres Siedepunktsminimum dẹjenigen Eckpunkt gegenüber liegt, der der Komponente mit dem niedrigsten Siedepunkt entspricht. Ferner kann dieser Fall bei Vorhandensein von ternären Extrema auftreten (Abb. 5).

Fall A bedeutet nach (25), daß

$$
\begin{aligned}
\beta_{13}^{\mathrm{r}}>0, & \alpha_{23}^{\mathrm{M}}>1, \\
\operatorname{oder} \beta_{13}^{\mathrm{M}}<0, & \alpha_{23}^{\mathrm{M}}<1 \quad \text { ist, daß also }
\end{aligned}
$$

im isobaren Diagramm bei einem binären Siedepunktsmaximum(minimum) die Steigung der besagten Schnittkurve in $\mathrm{M}$ negativ (positiv) ist (Abb.3 und 6). Welcher der Unterfälle von A auftritt, hängt von den Absolutwerten der Größen $\alpha_{23}^{\mathrm{M}}, \beta_{13}^{\mathrm{M}}$ und $x_{\mathrm{M}}$, also von der Steigung der erwähnten Schnittkurve in M, der Krümmung der 
binären Dampfdruckkurve in M und der Lage des Punktes M ab. Wir setzen nach dem Vorschlag von $\mathrm{Jost}^{1}$ (s. I, Gl. (10), in der mit $\alpha_{12}$ das Verhältnis $p_{1}^{0} / p_{2}^{0}$ bezeichnet ist) für das ternäre Gemisch die einfachen Beziehungen [s. V, Gln. (25 a) und (25b)] an:

$a_{13}=\frac{p_{1}^{0}}{p_{3}^{0}} \exp \frac{A_{13}\left(1-2 x_{1}-x_{2}\right)+\left(A_{12}-A_{23}\right) x_{2}}{R T}$,

$c_{23}=\frac{p_{2}^{0}}{p_{3}^{0}} \exp \frac{A_{23}\left(1-x_{1}-2 x_{2}\right)+\left(A_{12}-A_{13}\right) x_{1}}{R T}$,

wobei $A_{k i}$ gleich dem Vierfachen der auf $1 \mathrm{Mol}$ Mischung bezogenen maximalen zusätzlichen Freien Enthalpie (bei regulären Mischungen: der auf 1 Mol Mischung bezogenen maximalen Mischungswärme) für das binäre Gemisch $i, k$ ist.

Für das binäre Gemisch 1,3 folgt aus (31 a) mit $x_{2}=0$ [vgl. IV, Gl. (52)] :

$$
a_{13}=\frac{p_{1}^{0}}{p_{3}^{0}} \exp \frac{A_{13}\left(1-2 x_{1}\right)}{R T} .
$$

Hieraus ergibt sich:

$$
\beta_{13}=\beta_{13}^{\mathrm{M}}=\frac{\partial \ln \alpha_{13}}{\partial x_{1}}=-\frac{2 A_{13}}{R T} .
$$

Aus (31b) erhalten wir:

$$
a_{23}^{\mathrm{M}}=\frac{p_{2}^{0}}{p_{3}^{0}} \exp \frac{A_{23}\left(1-x_{\mathrm{M}}\right)+\left(A_{12}-A_{13}\right) x_{\mathrm{M}}}{K} \frac{1}{T} \text {. }
$$

Eine einfache Regel dafür, welche der verschiedenen Möglichkeiten des Falles A eintreten, läßt sich also auch bei Annahme der speziellen An. sätze (31) nicht geben, zumal $\alpha_{23}^{\mathrm{M}}$ wiederum von $x_{\mathrm{M}}$ abhängt.

In einem ternären azeotropen Punkt wird wegen $x_{1}{ }^{\prime}=x_{1}$ und $x_{2}{ }^{\prime}=x_{2}$ infolge von (4) und (5) $\alpha_{13}=1, \alpha_{23}=1$. Setzt man dem Vorigen entsprechende Entwicklungen für die Umgebung dieses Punktes an, so gelangt man - mit erheblich grö. ßerem mathematischen Aufwand als beim binären azeotropen Punkt - wieder zu Aussagen über den Verlauf der Destillationslinien. Van Dalfse $\mathrm{n}^{12}$ führte derartige Berechnungen durch. Wir geben diese nicht wieder, weil sie auf denselben allgemeingültigen Voraussetzungen wie die vorangehenden Betrachtungen beruhen und sich ebenfalls auf die isobare Destillation übertragen lassen. Die wichtigsten Ergebnisse der Rechnungen kann man folgendermaßen zusammenfassen. Es gibt bei jedem ternären azeotropen Punkt $\mathrm{P}$ (Ex tremum in der Dampfdruck- bzw. Siedefläche) zwei singuläre Kurven, die sich in $\mathrm{P}$ schneiden und in unmittelbarer Nähe von $\mathrm{P}$ geradlinig verlaufen. Im Falle eines Maximums oder Minimums wird eine dieser ausgezeichneten Kurven von allen übrigen Destillationslinien in der Nähe von $\mathrm{P}$ berührt (Abb. 5 bei $\mathrm{Mi}$ ). Im Falle eines Sattelpunktes verlaufen alle übrigen Destillationslinien in der Nähe von $\mathrm{P}$ hyperbolisch mit den geraden Stücken der singulären Kurven als Asymptoten (Abb. 6 bei S).

\section{d) Allgemeine Diskussion}

Sofern man besondere Komplikationen im mittleren Gebiet des Destillationsfeldes ausschließt, kann man mit den im Vorangehenden hergeleiteten Gesetzmäßigkeiten, die keinerlei Annahmen über die Natur der flüssigen Gemische enthalten, allein aus der Kenntnis der azeotropen Punkte und der Reihenfolge der Siedepunkte die Destillationsdiagramme in wesentlichen Zügen entwerfen. Van Dalfsen ${ }^{12}$ konstruierte eine Reihe solcher Diagramme. Soweit bis heute Messungen vorliegen, sind diese theoretischen Konstruktionen bestätigt worden.

Wir bringen als Beispiele von Destillations: diagrammen die Abb: 2 bis 6. (Mi bedeutet Minimum, Ma Maximum, S Sattelpunkt.)

Abb. 4 entspricht schematisch dem von Reinders und de Minjer ${ }^{\mathbf{1 3}}$ untersuchten System Benzol (A)Aceton (B)-Chloroform (C). Der in Abb. 6 dargestellte Fall dürfte (bis auf die willkürlich angenommenen Berührungsverhältnisse bei den drei binären azeotropen Punkten) schematisch das System Methanol(C)Aceton(B)-Chloroform (A) darstellen, bei dem der 1906 von van Dalfsen voreusgesagte Sattelpunkt von Ew ell und Welch ${ }^{\mathbf{1 4}} 1945$ entdeckt wurde.*

13 W. Re inders u. C. H. de Min je r, Recueil Trav. chim. Pays-Bas 59, 207, 369, 392 [1940].

is R. H. Ew ell u. L. M. W elch, Ind. Engng. Chem. 37, 1224 [1945].

* Für ternäre Siedepunktsminima, wie in Abb. 5, gibt es mehrere Beispiele, z. B. Methanol-AcetonCyclohexan. Fälle, bei denen ein ternärer Maximumsiedepunkt vorkommt, sind nicht bekannt.

Bei Systemen mit Mischungslücke, die, wie ÄthanolBenzol-Wasser, ein Gemisch mit tiefstem Siedepunkt aufweisen, handelt es sich nicht um einen ternären azeotropen Punkt, sondern um ein Temperaturminimum im heterogenen Gebiet, d. h. ein Minimum auf den räumlichen Dreiphasenkoexistenzkurven. Derartige Extrema (Minima oder Maxima) treten immer auf, wenin die drei Punkte, die den beiden flüssigen Schichten und dem Gleichgewichtsdampf entsprechen, 
Besondere Beachtung verdienen die schon erwähnten „Grenzlinien“. Im Falle von ternären azeotropen Punkten gibt es sogar mehr als zwei „Destillationsfelder" (Abb. 5 und 6). Ein Gemisch z. B., das durch einen Punkt in der Nähe von Mi in Abb. 5 dargestellt wird, kann je nach der Ausgangszusammensetzung bei fortgesetzter einfacher Destillation eine der drei reinen Komponenten oder eines der drei binären azeotropen Gemische als Rückstand geben. Man hat also in Abb. 5 drei Grenzlinien: $\mathrm{MiMi}_{1}, \mathrm{MiMi}_{2}$ und $\mathrm{MiMi}_{3}$.

Die ursprünglich von Ostwald ${ }^{15}$ vertretene Ansicht, alle Grenzlinien seien gerade und entsprächen relativen Extrema des Dampfdrucks, wurde eingehend von Schreinemakers ${ }^{16}$ und van Dalfse ${ }^{12}$ widerlegt. Da neuerdings Rein ders und de Minjer ${ }^{13}$ sowie Ewell und Welch ${ }^{14}$ bei einer Grenzlinie im isobaren Destillationsdiagramm von einem „Grat“ (ridge) bzw. ,.Tal“ (valley) in der Siedefläche (der Flüssigkeit) sprechen, ist es angebracht, auf die Frage der relativen Extrema noch einmal kurz einzugehen.

Schon van Dalfsen ${ }^{12}$ weist darauf hin, daß die Destillationslinien gar nichts zu tun haben mit relativen Maxima oder Minima, die man auf irgendwie definierten Schnittkurven erhält. Ferner kann man jeden beliebigen Punkt der Dampfdruck- bzw. Siedẹfläche zu einem Extremum auf einer Schnittkurve machen: Man legt durch den Punkt eine waagerechte Ebene, an die Schnittkurve mit der Dampfdruck- bzw. Siedefläche eine Tangente in diesem Punkt und durch diese Tangente eine senkrechte Ebene; die durch diese Ebene erzeugte Schnittkurve hat dann ein Extremum im betrachteten Punkt.

Wir untersuchen als Beispiel den geometrischen Ort für alle Extrema auf Schnittkurven in der Siedefläche, erzeugt durch Ebenen parallel zu der auf $A_{1} A_{3}$ (Abb. 1) lotrecht errichteten Ebene. Die Gleichung für diesen geometrischen Ort lautet:

$$
\partial T / \partial x_{1}=0 \text {. }
$$

(Forts. vonnebensteh. Fußnote)

auf einer Geraden im Darstellungsdreieck liegen ${ }^{3}$. Im Falle eines Minimums im heterogenen Gebiet ist es dann möglich (aber nicht notwendig), daß die betreffende Temperatur tiefer liegt als alle Siedepunkte im homogenen Gebiet. Ein Maximum im Entmischungsgebiet hingegen kann nie der höchste Siedepunkt des gesamten Systems sein. Von einem Minimum oder Sattelpunkt in der Siedefläche der Flüssigkeit und des Dampfes ${ }^{10}$ kann aber in diesen Fällen deshalb nicht die Rede sein, weil die Flüssigkeitssiedefläche im heterogenen Gebiet eine Regelfläche ist und die zugehörige räumliche Dampfkurve durch den Schnitt der beiden Teile der Dampfsiedefläche entsteht.

15 Wilh. Ostw ald, Abh. math.-physisch. Kl. sächs. Akad. Wiss. 25, 413 [1900]; Lehrbuch der Allgemeinen Chemie II, 2, S. 988-1017
Aus (2) kann man durch Elimination der $x_{i}$ und $\frac{\partial^{2} G}{\partial x_{i} \partial x_{k}}$ eine Differentialgleichung für die Variablen $P, T, x_{1}$ und $x_{2}$ ableiten [s. V, Gl. (36)]. Aus dieser ergibt sich mit (35):

$$
\begin{aligned}
\Phi \equiv\left(a_{13}-1\right)\left[1+x_{1}\left(1-x_{1}\right) \beta_{13}-x_{1} x_{2} \gamma_{13}\right] & \\
+\left(a_{23}-1\right)\left[x_{2}\left(1-x_{2}\right) \gamma_{13}-x_{1} x_{2} \beta_{13}\right] & =0 .
\end{aligned}
$$

Wir müssen also den Verlauf der Kurve $\Phi=0$ diskutieren. Für die drei Eckpunkte $A_{1}, A_{2}$ und $A_{3}$ erhalten wir: $\Phi=\alpha_{13}-1 \neq 0$. Da nun wegen IV, Gln. (32) - (35), bei Auftreten eines Extremums die Größe $\alpha_{i k}-1$ an den beiden Enden der binären Siedekurve entgegengesetztes Vorzeichen haben muß, hat $\alpha_{13}-1$ entweder in $A_{2}$ und $A_{1}$ oder in $A_{2}$ und $A_{3}$ verschiedene Zeichen, so daß die Kurve $\Phi=0$ entweder auf der Seite $A_{1} A_{2}$ oder auf der Seite $\mathrm{A}_{2} \mathrm{~A}_{3}$ endet.

Die Gleichung $\Phi=0$ wird ferner befriedigt durch: $\alpha_{1 ?}=1, x_{2}=0$ (Punkt M); $\alpha_{13}=1, \alpha_{23}=1$ (ternärer azeotroper Punkt).

Die Kurve, auf der die oben definierten relativen Extrema liegen, geht also vom binären azeotropen Punkt M aus, durch einen evtl. vorhandenen ternären azeotropen Punkt hindurch und endet auf einer der gegenüberliegenden Seiten. Wie aus (36) ersichtlich, ist diese Kurve im allgemeinen keine Gerade.

Hieraus geht zur Genüge hervor, daß Extrema auf bestimmten Schnittkurven nichts mit den Destillationslinien zu tun haben, und daß Extrema auf irgendwelchen Schnittkurven keinerlei mathematische oder physikalische Bedeutung haben.*

\section{Die Dampflinien}

Zur vollständigen Charakterisierung der. Verdampfungsgleichgewichte und der Destillationsfelder gehören außer den Destillationslinien die

16 F. A. H. Schreinemakers, Z. physik. Chem. 43, 671 [1903].

* Die Tatsache, daß nach den Befunden von Reinders und de Minjer ${ }^{\mathbf{1 3}}$ sowie Ewell und Welch ${ }^{\mathbf{1 4}}$ bestimmte Systeme, z. B. solche, wie sie in Abb. 4 u. 6 dargestellt sind, bei der Rektifikation ein grundsätzlich anderes Verhalten zeigen als die übrigen Systeme, darf daher nicht mit dem Auftreten eines "Grates“ in Zusammenhang gebracht werden. Entscheidend für die Rektifikation ist vielmehr der Umstand, daß bei diesen Systemen mit außergewöhnlichem Verhalten die Scharen der Destillationslinien von mehreren Punkten des Diagramms ausgehen (in Abb. 4 von B und $\mathrm{C}$, in Abb. 6 von $\mathrm{Mi}_{1}$ und $\mathrm{Mi}_{2}$ ), während in den übrigen Fällen (s. Abb. 2,3,5) alle Destillationslinien: von einem Punkte ausgehen. 
Scharen der „Dampflinien“, deren Definition oben gegeben wurde. In Abb. 2 ist die gestrichelte Linie BDA die der'Destillationslinie BFA entsprechende Dampflinie. Mit der Flüssigkeit $\mathrm{F}$ ist der Dampf D im Gleichgewicht.

Es folgt aus der Definition der Dampflinien, daß sie stets an der konvexen Seite der Destillationslinien verlaufen und bei denselben Punkten wie die Destillationslinien beginnen und enden; denn nur in den Eckpunkten des Darstellungsdreiecks und in azeotropen Punkten haben Flüssigkeit und Dampf gleiche Zusammensetzung.

Schnittpunkte von Dampflinien mit Destillations. linien treten nur auf, wenn die Destillationslinien Wendepunkte haben.

Aus (9) folgt:

$$
\frac{d^{2} x_{1}}{d x_{2}{ }^{2}}=\frac{d x_{2}{ }^{\prime}}{d x_{2}} \frac{d x_{1}^{\prime} / d x_{2}{ }^{\prime}-d x_{1} / d x_{2}}{x_{2}{ }^{\prime}-x_{2}} .
$$

Für $d^{2} x_{1} / d x_{2}{ }^{2}=0$ ergibt sich: $d x_{1}{ }^{\prime} / d x_{2}{ }^{\prime}$ $=d x_{1} / d x_{2}$, d. h. die Tangente an eine Destillationslinie im Wendepunkt ist gleichzeitig Tangente an die Dampfkurve im zugehörigen Dampfpunkt ${ }^{12}$.

Die Aufstellung einer Gleichung der Dampflinien gelingt nur, wenn die Gl. (15) integriert wird und $x_{1}$ und $x_{2}$ als Funktionen von $x_{1}{ }^{\prime}$ und $x_{2}{ }^{\prime}$ angegeben werden können. Ohne spezielle Ansätze für die $\alpha_{i k}$ ist das nicht möglich. Wir diskutieren im folgenden nur einige allgemeine Eigenschaften der Dampflinien.

Nach dem Henryschen Gesetz gilt für $x_{1} \rightarrow 0$, $x_{2} \rightarrow 0: x_{1}^{\prime} \rightarrow 0, \quad x_{2}^{\prime} \rightarrow 0$. Wir erhalten daher aus (4), (5), (17) und (18) für die Nähe eines Eckpunktes:

$$
x_{1}=x_{1}{ }^{\prime} / \alpha_{13}^{0}, x_{2}=x_{2}{ }^{\prime} / \alpha_{23}^{0} .
$$

Einsetzen in (19) ergibt:

$$
x_{1}^{\prime}=C^{\prime} x_{2}^{\prime} \frac{c_{13}^{0}-1}{c_{23}^{0}-1} .
$$

Die Dampflinien zeigen demnach in der Nähe von Eckpunkten dasselbe Verhalten wie die zugehörigen Destillationslinien.

Für die Umgebung eines binären azeotropen Punktes M setzen wir an:

$$
\begin{aligned}
& x_{1}=x_{\mathrm{M}}+\xi, \quad x_{1}{ }^{\prime}=x_{\mathrm{M}}+\xi^{\prime}, \\
& x_{2}=\eta_{1}, \quad x_{2}{ }^{\prime}=\eta^{\prime} .
\end{aligned}
$$

Wie schon Lorentz und Schreinemakers ${ }^{11}$ bei der Diskussion der Verdampfungs- und Kondensationskurven gezeigt haben, verschwinden $\xi$ und $\xi^{\prime}$ sowie $\eta$ und $\eta^{\prime}$ jeweils von derselben Ordnung:

$$
\begin{aligned}
& \xi^{\prime}=c \xi, \quad\left(c \text { und } c^{\prime} \text { endlich }\right) \\
& \eta^{\prime}=c^{\prime} \eta .
\end{aligned}
$$

Die letzte Beziehung kann man sofort herleiten aus dem Henryschen Gesetz im weiteren Sinne [s. V, Gl. (31)] :

$$
p_{2}=P x_{2}{ }^{\prime}=K x_{2}\left(\text { für } x_{2} \rightarrow 0\right),
$$

worin $K$ von $x_{\mathbf{M}}$ abhängt.

Daher bleibt die Form der Gln. (26) und (27) erhalten, wenn man $\xi^{\prime}$ und $\eta^{\prime}$ einführt. Die Dampflinien zeigen also auch in der Nähe von binären azeotropen Punkten dasselbe Verhalten wie die zugehörigen Destillationslinien. Entsprechendes kann man für ternäre azeotrope Punkte ableiten.

Es ist nun ohne weiteres ersichtlich, wie in den Abb. 3 bis 6 die Dampflinien einzuzeichnen sind.

Es ergibt sich aus unserer Diskussion der Destillationslinien, daß die von der holländischen Schule aufgestellten Gesetzmäßigkeiten insofern allgemeingültig sind, als sie für die flüssige Phase nur die universellen Grenzgesetze für unendliche Verdünnung enthalten, andererseits aber insofern wieder speziell sind, als sie voraussetzen, daß der Dampf sich wie ein ideales Gasgemisch verhalte. Gibt man die letzte Annahme auf, so bedingt dies keine schwerwiegenden Änderungen, da bei hinreichend geringer Konzentration zweier Komponenten im Gas die Fugazitäten stets in die Partialdrucke der betreffenden Komponenten übergehen; lediglich die Diskussion für die azeotropen Punkte müßte modifiziert werden. Ferner erkennt man, daß alle Gesetzmäßigkeiten nicht nư für den isothermen, sondern auch für den isobaren Fall Gültigkeit besitzen. Dies war bisher nicht bewiesen worden. Des weiteren zeigt die kurze analytische Diskussion der Dampflinien, daß für diese ebenfalls einfache allgemeine Gesetze gelten.

Hrn. Prof. W. J o s t danke ich für wertvolle Kritik und dafür, daß er mir die Durchführung dieser Arbeit in seinem Institut ermöglicht hat. 